\title{
Evaluation of copy number variation and gene expression in neurofibromatosis type-1-associated malignant peripheral nerve sheath tumours
}

\author{
Laura E Thomas ${ }^{1}$, Jincy Winston ${ }^{1}$, Ellie Rad ${ }^{1}$, Matthew Mort ${ }^{1}$, Kayleigh M Dodd ${ }^{1}$, Andrew R Tee ${ }^{1}$, \\ Fionnuala McDyer ${ }^{2}$, Stephen Moore ${ }^{2}$, David N Cooper ${ }^{1}$ and Meena Upadhyaya ${ }^{1 *}$
}

\begin{abstract}
Background: Neurofibromatosis type-1 (NF1) is a complex neurogenetic disorder characterised by the development of benign and malignant tumours of the peripheral nerve sheath (MPNSTs). Whilst biallelic NF1 gene inactivation contributes to benign tumour formation, additional cellular changes in gene structure and/or expression are required to induce malignant transformation. Although few molecular profiling studies have been performed on the process of progression of pre-existing plexiform neurofibromas to MPNSTs, the integrated analysis of copy number alterations (CNAs) and gene expression is likely to be key to understanding the molecular mechanisms underlying NF1-MPNST tumorigenesis. In a pilot study, we employed this approach to identify genes differentially expressed between benign and malignant NF1 tumours.
\end{abstract}

Results: SPP1 (osteopontin) was the most differentially expressed gene (85-fold increase in expression), compared to benign plexiform neurofibromas. Short hairpin RNA (shRNA) knockdown of SPP1 in NF1-MPNST cells reduced tumour spheroid size, wound healing and invasion in four different MPNST cell lines. Seventy-six genes were found to exhibit concordance between CNA and gene expression level.

Conclusions: Pathway analysis of these genes suggested that glutathione metabolism and Wnt signalling may be specifically involved in NF1-MPNST development. SPP1 is associated with malignant transformation in NF1-associated MPNSTs and could prove to be an important target for therapeutic intervention.

Keywords: NF1, MPNSTs, Copy number alterations, Gene expression profiling, SPP1

\section{Background}

Neurofibromatosis type 1 (NF1) (MIM\# 162200), a complex autosomal dominant disorder with a highly variable clinical phenotype, affects approximately 1 in 4,000 people worldwide (Huson, 2008). Malignant complications are relatively rare manifestations of this disorder but include brain tumours, optic gliomas and malignant peripheral nerve sheath tumours (MPNSTs) [1,2]. MPNSTs can either occur sporadically or instead may develop from the malignant transformation of pre-existing NF1associated plexiform neurofibromas (PNF) or from a focal

\footnotetext{
* Correspondence: upadhyaya@cardiff.ac.uk

'Institute of Medical Genetics, School of Medicine, Cardiff University, Cardiff CF14 4XN, UK

Full list of author information is available at the end of the article
}

subcutaneous neurofibroma. The lifetime risk of developing MPNSTs in NF1 patients is $10 \%-15 \%[3,4]$.

MPNSTs are a significant cause of morbidity and mortality in NF1. Upon first presentation, MPNSTs are often non-resectable because the tumour is already at a late stage of development; metastases may also be present, most commonly in the lung but also in the liver and brain. Consequently, the 5-year survival rate for individuals with these tumours is only $20 \%-50 \%$, and the 10 year survival rate is even lower (7.5\%) [5]. Currently, there are no effective treatments for MPNSTs; although complete surgical excision with clear margins is the therapy of choice, chemotherapy has sometimes also been employed despite its limited effectiveness. Several risk factors confer an increased likelihood of MPNST development, e.g. multiple internal PNFs [6], previous radiation 
therapy, the presence of an inherited gross genomic deletion that removes the entire NF1 gene [7], the presence of neurofibromatous neuropathy [8] or a family history of NF1-MPNSTs [9]. Since there are no reliable predictive or prognostic biomarkers for MPNSTs, the progression of a pre-existing plexiform neurofibroma to an MPNST cannot currently be predicted in advance on the basis of molecular (copy number and/or gene expression) profiling despite recent significant advances in this field. Beert et al. (2011) demonstrated recurrent homozygous loss of the CDKN2A locus in 15/16 atypical neurofibromas; this finding supports the notion that atypical neurofibromas constitute intermediates between benign neurofibroma and MPNSTs and further suggests that CDKN2A loss is an early step in the progression of neurofibroma to MPNSTs [10]. A detailed knowledge of the NF1 somatic mutational spectrum of MPNSTs is, however, a prerequisite for the development of targeted therapies. Although somatic inactivation of the wild-type NF1 allele is presumed to be the key step in NF1-associated tumour development, this cannot on its own explain the malignant transformation of benign plexiform neurofibromas to MPNSTs. This indicates that additional genetic (and potentially epigenetic) alterations are required to bring about malignancy.

High-throughput whole genome microarray profiling has proved to be one of the most effective methods to analyse large numbers of clinical samples across multiple tumour types. Indeed, a considerable number of studies have identified somatic copy number alterations (CNA) and concomitant gene expression changes in benign and malignant NF1-associated tumours [11-30]. However, despite the plethora of previous array-based investigations, relatively few genes have so far been identified that are consistently mutated across multiple MPNSTs. Such frequently mutated genes may harbour 'driver mutations' (as opposed to 'passenger mutations') that promote NF1 malignant transformation. MPNST development is clearly a complex multistep process in which the mutation of a large number of genes, contributing to the deregulation of multiple signalling and regulatory pathways, is to be expected. Whole genome analysis and, in particular, the focussed investigation of specific pathways are likely to be key to determining the underlying molecular mechanisms involved in MPNST tumorigenesis. Genes that could be informative in a prognostic context may be identified through the use of integrated $\mathrm{CNA}$ /gene expression analysis performed on the same sample sets $[31,32]$. To this end, the present study employed Affymetrix Human Exon 1.0 ST Arrays to screen both PNF and MPNST tumour DNAs from nine unrelated NF1 patients to generate data on differences in gene expression at the whole genome level between benign and malignant tumours with which to compare with previously generated CNA data derived from the same tumours in order to disclose novel genes and pathways that could be important in MPNST development [25].

\section{Results}

Identification of differentially expressed genes between neurofibromas and MPNSTs

For differential expression analysis, different sets of genes were identified from the various analyses (Figure 1a,b) that we performed: (1) 4 benign tumour samples vs. 5 malignant tumour samples with stringent criteria applied, (2) 4 benign tumour samples vs. 5 malignant tumour samples under less stringent criteria, (3) 3 benign tumour samples vs. 3 malignant tumour samples under stringent criteria and (4) 3 benign tumour samples vs. 3 malignant tumour samples under less stringent criteria (Additional file 1: Table S2). The genes identified employing the less stringent criteria from the 4 vs. 5 analyses were crosscompared with the corresponding list of genes identified by means of the 3 vs. 3 analysis (Figure 1a). The same process was then followed for those genes identified using the more stringent criteria (Figure 1b). Given the relatively

$\mathrm{a}$ 4 benign vs. 5 malignant $\quad 3$ benign vs. 3 malignant

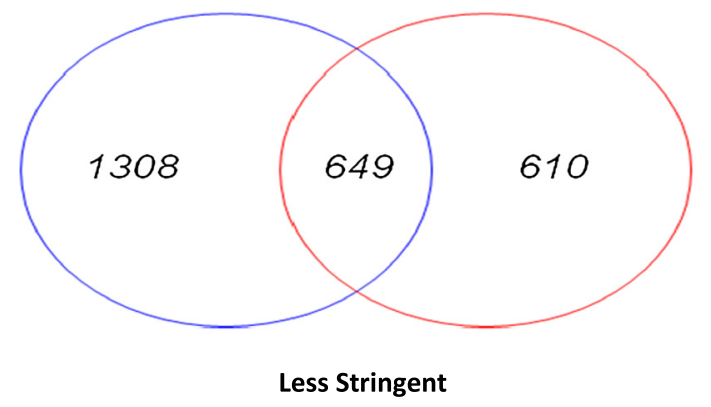

b 4 benign vs. 5 malignant 3 benign vs. 3 malignant

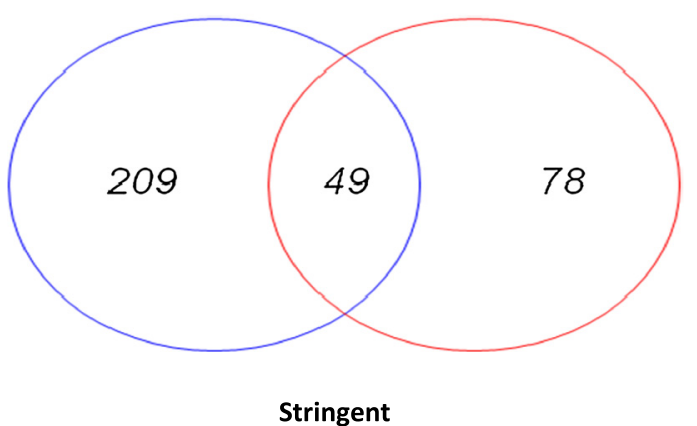

Figure 1 Venn diagram comparison based upon the less stringent and stringent gene list. a. Venn diagram based upon the less stringent gene lists from the 4 vs. 5 and 3 vs. 3 pairwise comparisons. b. Venn diagram based upon the stringent gene lists from the 4 vs. 5 and 3 vs. 3 pairwise comparisons. 
small number of patients included in this study, the presence of outliers (patients exhibiting variable expression compared with the median) was expected to have a considerable influence on the combined results. With this in mind, the analysis as described above was performed with and without those outlier/variable samples.

The number of genes identified as being differentially expressed between benign and malignant tumours under more or less stringent criteria in the 3 vs. 3 and 4 vs. 5 analyses can be found in Table 1. Under the less stringent criteria, statistical testing employed a $P$ value threshold of 0.05 , which served to identify genes displaying $\geq 1$.3-fold (absolute) differential gene expression. In the differential analysis, which considered all profiled samples (4 benign vs. 5 malignant), a total of 1,957 genes were identified as being differentially expressed between benign and malignant tumours whereas in the differential analysis which excluded the three samples at the expression range extremes ( 3 benign vs. 3 malignant), only 1,259 genes were identified. The two different conditions of stringency were used for the sake of comparison. However, for the final analysis, the genes identified under the more stringent criteria were used. Under the more stringent criteria, statistical testing employed a $P$ value threshold of 0.005 , which was deemed to be sufficient to identify genes displaying 1.5-fold or more (absolute) differential gene expression. In the 4 vs. 5 stringent gene list analysis, 258 genes were identified as being differentially expressed between benign and malignant tumours whereas in the 3 vs. 3 analyses, the number of genes identified was 127 . The top 20 significantly differentially expressed genes identified using the more stringent criteria are given in Table 2.

The SPP1 gene [NCBI: NM_001040058] (osteopontin; OPN) was found to exhibit the most significantly elevated differential mean expression level between the benign and malignant tumours. SPP1 was therefore selected for further functional analysis to assess its possible role in malignant transformation. Exon array analysis identified a mean expression level of $12.06(\log 2)$ for SPP1 in malignant tumours and a mean expression level of $5.65(\log 2)$ in benign plexiform neurofibromas. This equates to an 85 -fold increase (based on anti-logged LS means) in expression in

Table 1 Definitions of stringent and less stringent analytical criteria and the numbers of genes detected by the 3 vs. 3 and 4 vs. 5 analyses under both sets of criteria

\begin{tabular}{lll}
\hline & Less stringent criteria & Stringent criteria \\
\hline Statistical testing $P$ value & 0.05 & 0.005 \\
Fold change & 1.3 & 1.5 \\
Number of genes 4 vs. 5 & 1,957 & 258 \\
Number of genes 3 vs. 3 & 1,259 & 127 \\
\hline
\end{tabular}

Definition of stringent and less stringent analytical criteria and the numbers of genes detected by the t vs. 3 and 4 vs. 5 analyses under both sets of criteria.
MPNSTs compared to benign plexiform neurofibromas $(P=0.0002)$ (Table 2). Since this was the highest fold change in expression observed between benign and malignant tumours for all the genes identified in this analysis, an association with malignant transformation was strongly suspected. Significant reductions in the expression of $A D H 1 B$ and GRIK2 ( $P$ values 0.0003 and 0.0004 , respectively) in MPNSTs as compared to benign plexiform neurofibromas were however also observed (Table 2).

\section{Quantitative real-time PCR (q-PCR)}

Gene expression changes initially detected by Affymetrix Human Exon 1.0 ST Array analysis were validated by q-PCR. We aimed to determine where there was a correlation between the presence of a copy number alteration and gene expression (either a concomitant increase in copy number and gene expression or a decrease in both) as assessed by quantitative PCR. This was determined by identifying whether the same directional change as noted above was identified in the array data from the current study and CNA data from the previous analyses [25] (Additional file 2: Table S3). A correlation was noted for 11 of the 20 differentially expressed genes analysed (Additional file 3: Figure S1) (ADH1B, FAP, FLJ42200, GRIK2, GTDC1, KIAA0746, KRTAP13-4, PEG3, NSBP1, $P T G I S$ and SPP1). For six of the genes (CCT5, CD72, COL10A1, FAM177A1, SLC25A12 and TDP1), we were unable to identify a correlation (i.e. concordance) between the copy number alteration and gene expression in six of the tumour samples tested. For the remaining three genes (CSRP1, GRIK3 and EFHC2), half the samples yielded findings compatible with, and anticipated from, the results of the exon array experiments; the remaining samples either demonstrated no change in gene expression or the opposite change to that anticipated on the basis of the exon array results (i.e. a decrease in gene expression identified by array, increase in gene expression identified by Q-PCR) (see Additional file 3: Figure S1).

\section{Integration of copy number analysis with differentially expressed genes}

CNA data were derived from a previously published analysis of the same tumours [25]. In total, 27 genes were common to HMM and expression analysis, whereas 120 genes were common to segmentation and expression analysis. We then selected those genes that were common to expression analysis and HMM and/or segmentation analysis (121 genes in total; see Figure 2). Of these 121 genes, gene expression and CNA data were concordant in $76(63 \%)$ cases, with all 76 genes displaying the same directional change of both CNA and gene expression (Additional file 2: Table S3). The 76 genes that were concordant for copy number and gene expression 
Table 2 The top $\mathbf{2 0}$ genes identified as being significantly differentially expressed between MPNSTs and PNFs in the more stringent analysis of 3 vs. 3 samples

\begin{tabular}{|c|c|c|c|c|c|c|c|c|c|}
\hline \multirow[t]{2}{*}{ Transcript ID } & \multirow[t]{2}{*}{ Gene symbol } & \multirow[t]{2}{*}{ Ref seq } & \multirow{2}{*}{$\begin{array}{l}\text { Mean } \\
\text { expression } \\
\text { Benign }\end{array}$} & \multirow{2}{*}{$\begin{array}{l}\text { Mean } \\
\text { expression } \\
\text { Malignant }\end{array}$} & \multirow{2}{*}{$\begin{array}{l}\text { Fold change } \\
\text { Benign vs. } \\
\text { malignant }\end{array}$} & \multirow{2}{*}{$\begin{array}{l}P \text { value } \\
\text { Benign vs. } \\
\text { malignant }\end{array}$} & \multirow[t]{2}{*}{ Chromosome } & \multirow[t]{2}{*}{ Start } & \multirow[t]{2}{*}{ Stop } \\
\hline & & & & & & & & & \\
\hline 2584134 & FAP & NM_004460 & 4.87 & 8.75 & 14.75 & 0.0001 & 2 & 163027200 & 163101589 \\
\hline 4013828 & NSBP1 & NM_030763 & 5.22 & 3.67 & -2.92 & 0.0001 & $x$ & 80211860 & 80457431 \\
\hline 3917555 & KRTAP13n4 & NM_181600 & 3.77 & 3.13 & -1.55 & 0.0001 & 21 & 31797943 & 31809269 \\
\hline 3908934 & PTGIS & NM_000961 & 4.58 & 6.60 & 4.06 & 0.0002 & 20 & 48120414 & 48185550 \\
\hline 2764192 & KIAA0746 & NM_015187 & 4.33 & 5.82 & 2.80 & 0.0002 & 4 & 25722248 & 25865344 \\
\hline 2735027 & SPP1 & NM_001040058 & 5.65 & 12.06 & 85.19 & 0.0002 & 4 & 88408243 & 88904561 \\
\hline 3532353 & FAM177A1 & NM_001079519 & 8.01 & 7.11 & -1.86 & 0.0003 & 14 & 35508201 & 35582327 \\
\hline 2779231 & $A D H 1 B$ & NM_000668 & 6.50 & 3.18 & -9.99 & 0.0003 & 4 & 100226131 & 100242895 \\
\hline 2406926 & GRIK3 & NM_000831 & 7.20 & 3.85 & -10.19 & 0.0004 & 1 & 37266614 & 37524753 \\
\hline 3916290 & FLJ42200 & AK124194 & 3.32 & 4.05 & 1.65 & 0.0004 & 21 & 25353415 & 25920256 \\
\hline 2970942 & COL10A1 & NM_000493 & 3.56 & 4.67 & 2.17 & 0.0004 & 6 & 116440092 & 116518549 \\
\hline 2450865 & CSRP1 & NM_004078 & 9.09 & 7.28 & -3.50 & 0.0005 & 1 & 201445919 & 201481500 \\
\hline 2918982 & GRIK2 & NM_175768 & 7.02 & 3.47 & -11.66 & 0.0005 & 6 & 101841683 & 102690448 \\
\hline 3204648 & CD72 & NM_001782 & 4.08 & 5.08 & 1.99 & 0.0005 & 9 & 35609970 & 35646790 \\
\hline 4006326 & EFHC2 & NM_025184 & 4.84 & 3.42 & -2.67 & 0.0005 & $x$ & 44006888 & 44309044 \\
\hline 2586845 & SLC25A12 & NM_003705 & 5.56 & 6.30 & 1.68 & 0.0006 & 2 & 172639117 & 172750960 \\
\hline 2579439 & GTDC1 & NM_001006636 & 6.95 & 5.92 & -2.05 & 0.0006 & 2 & 144702190 & 145123135 \\
\hline 3872053 & PEG3 & NM_006210 & 5.58 & 4.17 & -2.65 & 0.0006 & 19 & 57285930 & 57352082 \\
\hline 2801526 & CCT5 & NM_012073 & 8.62 & 9.33 & 1.64 & 0.0006 & 5 & 10250111 & 10266501 \\
\hline 3548152 & TDP1 & NM_018319 & 4.17 & 5.49 & 2.50 & 0.0007 & 14 & 90421293 & 90511103 \\
\hline
\end{tabular}

The top 20 genes identified as being significantly differentially expressed between MPNSTs and PNFs in the more stringent analysis of 3 vs. 3 samples.

changes were regarded as potential candidates for involvement in malignancy (Additional file 2: Table S3).

\section{Pathway analysis}

The 76 concordant genes, which were common to both the CNA and expression analyses, were interrogated using all pathways in the METACORE database. Pathways in which more than one gene was subject to concomitant alterations in both copy number and expression were then listed. The top ten pathways (by $P$ value) are given in Table 3 . These pathways include the glutathione metabolism, Wnt signalling, cell adhesion and alpha-6/ beta 4 integrin pathways.

\section{SPP1 knockdown in multiple MPNST cell lines impairs} tumour formation, wound healing and invasion

Given that we observed significant differences in the expression level of SPP1 in MPNSTs as compared with the benign plexiform neurofibromas and the fact that SPP1 plays significant roles in both tumorigenesis and metastasis [33], we knocked down expression of the SPP1 gene in four MPNST cell lines (ST8814, S462, S1844.1 and
S1507.2) and examined both the ability of these cell lines to form tumours (Figure 3a) and the effect of knockdown on wound healing and invasion. Both control and SPP1 knockdown cell lines formed tumour colonies in soft agar. However, SPP1 knockdown caused a significant reduction in tumour spheroid size $(n=40, P=$ 0.0001 ) in all MPNST cell lines tested (Figure $3 \mathrm{~b})$. We next analysed the effects of SPP1 knockdown on cell migration during wound healing (Figure 4a). In all cell lines, knockdown of SPP1 robustly inhibited wound closure (Figure 4b) ( $P$ values for the ST8814, S462, S1844.1 and S1507.2 cell line were 0.03, 0.001, 0.001 and 0.003, respectively), suggesting a possible role for SPP1 in metastasis. This role was further supported by the results of cellular invasion assays, which revealed that SPP1 knockdown significantly reduces the cells' invasive properties ( $P$ values for the ST8814, S462, S1844.1 and S1507.2 cell line were $0.0001,0.001,0.0001$ and 0.001 , respectively) (Figure $4 \mathrm{~b}, \mathrm{c}$ ). Validation of specific gene knockdown using short hairpin RNA (shRNA) clones was completed by Western blot analysis. A $\beta$-actin control blot was performed to confirm that there were no 


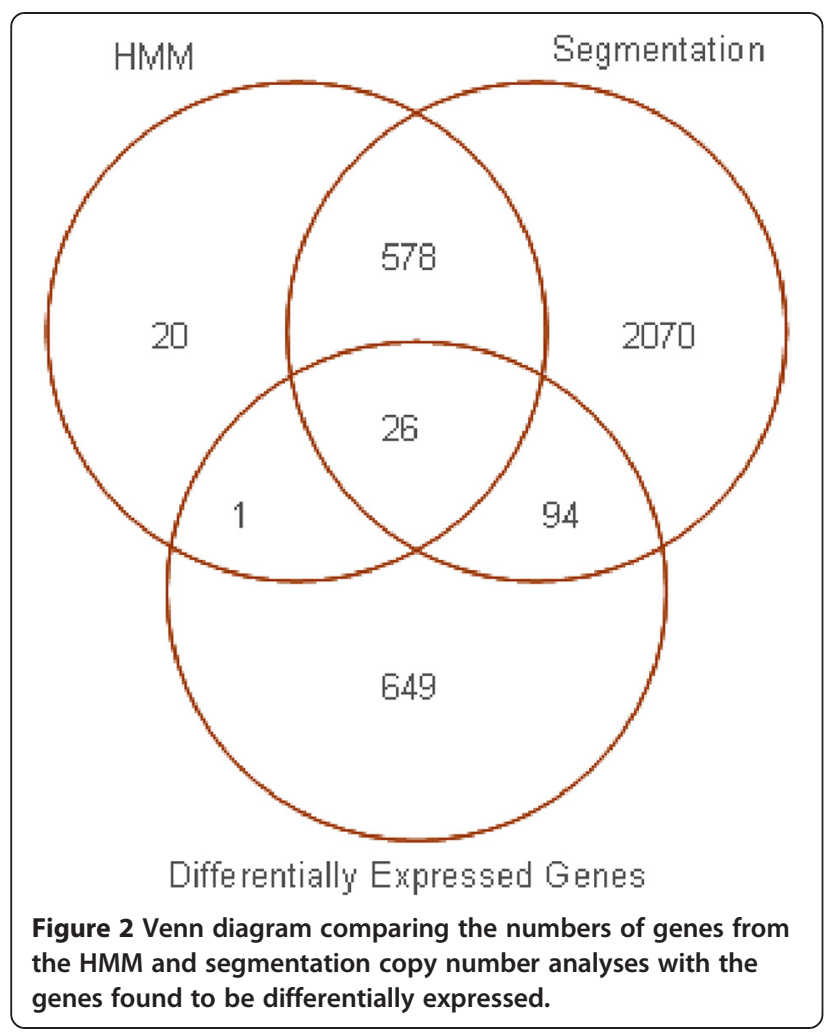

shRNA off-target effects (Figure 5). In summary, SPP1 knockdown with shRNA resulted in a significant reduction in both wound healing and invasiveness.

\section{Discussion}

The functional loss of neurofibromin, due to NF1 gene inactivation, leads to increased cell growth and proliferation through constitutive Ras pathway signalling. Although this is necessary for benign neurofibroma formation, it is insufficient to explain the malignant transformation of a benign PNF to an MPNST, since additional genetic lesions (and/or epigenetic modifications) are required for this to occur [25].

Initially, this study aimed to identify genes that contribute to the malignant transformation of benign neurofibromas by establishing which genes were differentially expressed between benign and malignant tumours in NF1 patients. A previously compiled list of genes located within regions encompassed by copy number alterations (CNAs) identified in the NF1-MPNSTs which were also used in the current study [25] was then cross-compared with a list of genes differentially expressed in the same NF1-MPNST samples, which were analysed for this study through Affymetrix exon array analysis. Finally, an additional study involving pathway analysis was performed on those genes that were common to the results of the CNA and exon array analyses.
In previous studies of NF1-MPNSTs, many genetic alterations, including copy number alterations and differential gene expression, have been identified but no specific molecular signature pathognomonic of malignant transformation has so far been defined [11-30]. Identifying genes that are differentially expressed between benign and malignant tumours not only promises to improve our understanding of the process of malignant transformation but should also aid in identifying potential therapeutic targets.

This study employed the GeneChip Human Gene 1.0 ST array (Affymetrix, Santa Clara, USA). This array is a whole transcript-based array for gene expression profiling which, unlike older Affymetrix arrays, queries the entire transcript instead of just the $3^{\prime}$ end [34]. Additionally, this array uses a labelling protocol that generates biotinylated sense strand DNA instead of complementary RNA (cRNA), thereby yielding DNA-DNA duplexes, which are more specific than the RNA-DNA duplexes generated using standard protocols [35]. This array provides approximately 25 -mer probes designed to be distributed across the transcribed regions of each gene with a median of 26 probes per gene, giving two complementary levels of expression analysis in a single experiment, both "exon-level" and "gene-level" analysis. The array interrogates 28,869 well-annotated genes with 764,885 distinct probes. The array is based on the March 2006 (UCSChg18, NCBI Build 36) human genome sequence assembly. The Affymetrix Human Exon 1.0 ST array contains 17,881 transcripts. Whole genome Affymetrix exon arrays have been employed in a number of different studies of cancer including non-small cell lung, colon, bladder and prostate cancer [36,37].

The comparative transcriptome analysis reported here identified the SPP1 gene to be the single most differentially overexpressed gene in NF1-MPNSTs as compared to benign tumours. SPP1 was selected for further study not only for this reason but also because of its welldocumented involvement in cell signalling, tumorigenesis and metastasis [38-49]. shRNA knockdown of four different MPNST cell lines revealed a significant reduction in tumour colony size growth, wound healing and cell invasion, thereby supporting a role for the increased expression of SPP1 in the malignant transformation and invasion of cells during NF1-MPNST development. SPP1 is an extracellular matrix protein with cytokine properties. It is involved in extracellular matrix (ECM) and adhesionrelated pathways where it performs key roles in cell-cell communication, focal adhesion, immune cell activation and immune cell migration. It plays an essential role in the pathway that leads to type I immunity, thereby enhancing the production of interferon-gamma and interleukin12 and reducing interleukin-1 synthesis. In terms of an association with cancer, SPP1 has been shown to 
Table 3 Pathway Enrichment analysis

\begin{tabular}{|c|c|c|c|c|c|}
\hline & Pathway name & $P$ value & Genes & $\begin{array}{l}\text { Fold change ( } 3 \text { benign } \\
\text { vs. } 3 \text { malignant) }\end{array}$ & $\begin{array}{l}\text { Fold change ( } 4 \text { benign } \\
\text { vs. } 5 \text { malignant }\end{array}$ \\
\hline \multirow[t]{2}{*}{1} & \multirow[t]{3}{*}{ Glutathione metabolism } & \multirow[t]{3}{*}{0.00056} & GSTM1 & -3.25 & -2.27 \\
\hline & & & GSTM2 & -3.25 & -2.27 \\
\hline & & & GSTM4 & -3.25 & -2.27 \\
\hline \multirow[t]{3}{*}{2} & \multirow[t]{3}{*}{ Glutathione metabolism/human version } & \multirow[t]{3}{*}{0.00059} & GSTM1 & -3.25 & -2.27 \\
\hline & & & GSTM2 & -3.25 & -2.27 \\
\hline & & & GSTM4 & -3.25 & -2.27 \\
\hline \multirow[t]{3}{*}{3} & \multirow[t]{3}{*}{ Glutathione metabolism/rodent version } & \multirow[t]{3}{*}{0.00073} & GSTM1 & -3.25 & -2.27 \\
\hline & & & GSTM2 & -3.25 & -2.27 \\
\hline & & & GSTM4 & -3.25 & -2.27 \\
\hline \multirow[t]{2}{*}{4} & \multirow{2}{*}{$\begin{array}{l}\text { Development: Wnt signalling pathway. Degradation of } \\
\text { beta-catenin in the absence Wnt signalling }\end{array}$} & \multirow[t]{2}{*}{0.00106} & CSNK1D & 2.42 & 1.99 \\
\hline & & & $D A B 2$ & 3.46 & 2.65 \\
\hline \multirow[t]{2}{*}{5} & \multirow[t]{2}{*}{ Cell adhesion: PLAU signalling } & \multirow[t]{2}{*}{0.00445} & $H G F$ & 7.80 & 10.97 \\
\hline & & & SERPINE1 & 5.74 & 4.54 \\
\hline \multirow[t]{2}{*}{6} & \multirow[t]{2}{*}{ Role of alpha- $6 /$ beta-4 integrins in carcinoma progression } & \multirow[t]{2}{*}{0.00589} & HGF & 7.80 & 10.97 \\
\hline & & & LIMK1 & 2.04 & 2.23 \\
\hline \multirow[t]{2}{*}{7} & \multirow[t]{2}{*}{ Development: TGF-beta-dependent induction of EMT via MAPK } & \multirow[t]{2}{*}{0.00641} & $D A B 2$ & 3.46 & 2.65 \\
\hline & & & SERPINE1 & 5.74 & 4.54 \\
\hline \multirow[t]{2}{*}{8} & \multirow[t]{2}{*}{ Transport: macropinocytosis regulation by growth factors } & \multirow[t]{2}{*}{0.01130} & $H G F$ & 7.80 & 10.97 \\
\hline & & & LIMK1 & 2.04 & 2.23 \\
\hline \multirow[t]{2}{*}{9} & \multirow{2}{*}{$\begin{array}{l}\text { Development: regulation of epithelial-to-mesenchymal } \\
\text { transition (EMT) }\end{array}$} & \multirow[t]{2}{*}{0.01165} & HGF & 7.80 & 10.97 \\
\hline & & & SERPINE1 & 5.74 & 4.54 \\
\hline \multirow[t]{2}{*}{10} & \multirow[t]{2}{*}{ Transport: clathrin-coated vesicle cycle } & \multirow[t]{2}{*}{0.01421} & PREB & 1.45 & 1.43 \\
\hline & & & $D A B 2$ & 3.46 & 2.65 \\
\hline
\end{tabular}

The pathways were ranked by hypergeometric $P$ values, and a summary of the top ten pathways is reported. The $P$ value represents the probability that a gene set of this size would co-occur by chance alone. Network objects represent the ratio of the number of genes from the list compared to the total number of genes known to be associated with the pathway.

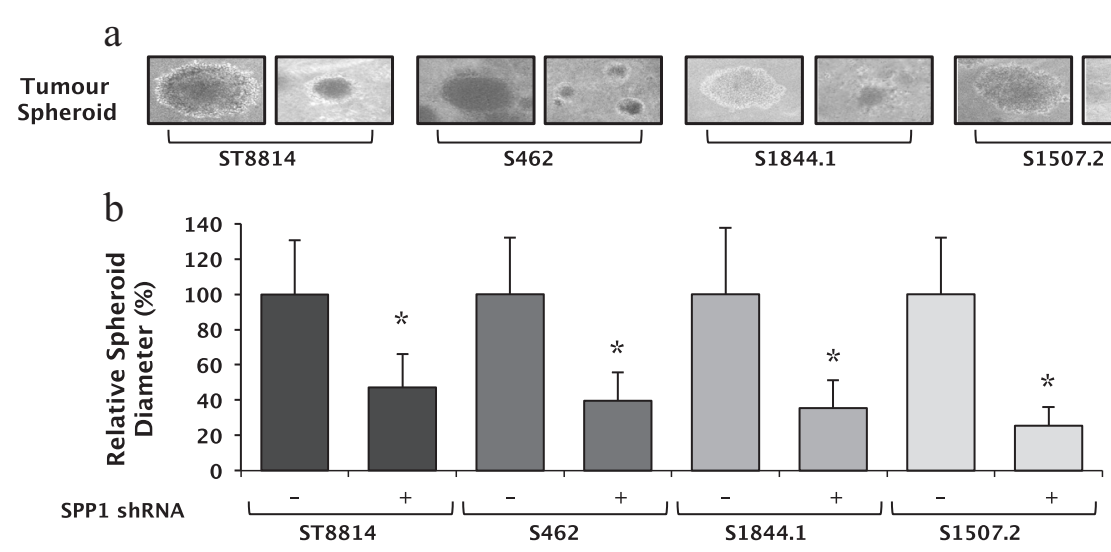

Figure 3 SPP1 knockdown reduces tumour size in soft agar. Stable ST8814, S462, S1844.1 and S1507.2 cell lines, expressing either non-target or SPP1 shRNA as indicated, were subjected to tumour spheroid growth assays. a) Photographs of 40 tumours were taken after 2 weeks of incubation. The diameter of all tumours was measured using ImageJ software; the scale bar on images represents $250 \mu \mathrm{m}$. b) The percentage of tumour size was calculated from the 40 tumours for both the control and SPP1 shRNA. SPP1 knockdown significantly reduced tumour size in all four MPNST cell lines, consistent with a role for SPP1 in tumour growth $(P=0.0001)$, ${ }^{*} P<0.05$ when comparing treated vs. untreated cells. 

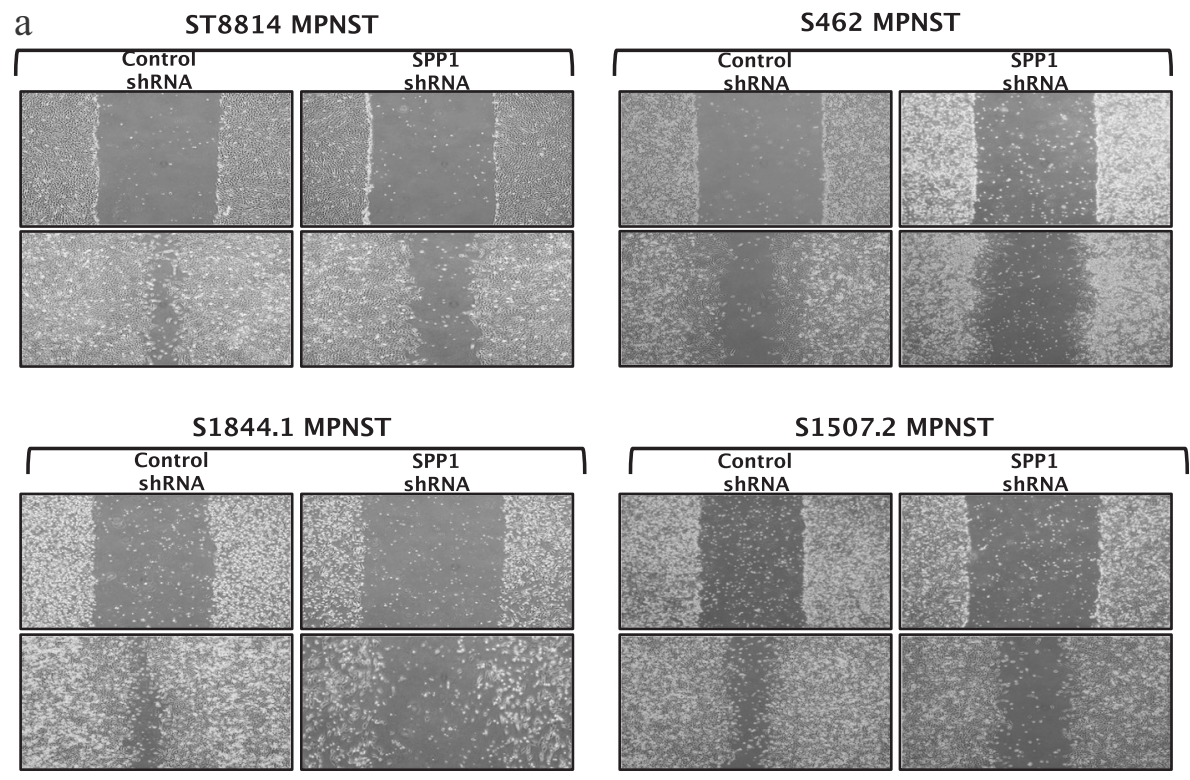

b
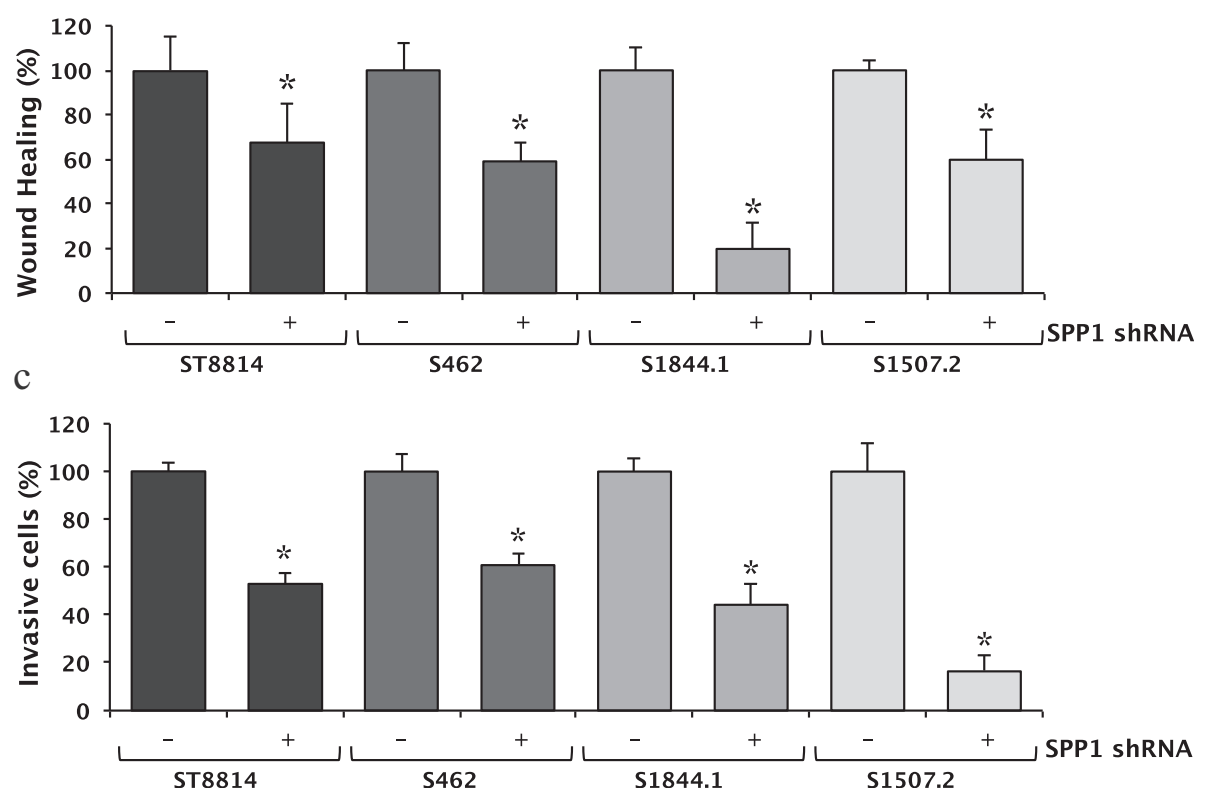

Figure 4 SPP1 knockdown impairs wound healing and cell invasion. Stable ST8814, S462, S1844.1 and S1507.2 cell lines, expressing either non-target or SPP1 shRNA, as indicated, were subjected to a wound healing assay. a) Pictures of cells were taken at 0 and $18 \mathrm{~h}$ after wounding. b) The percentage of cell migration was calculated using three individual experiments. SPP1 knockdown significantly reduced cell migration during wound healing in all four MPNST cell lines [P values for the four cell lines were as follows: ST8814 (0.03), S462 (0.001), S1844.1 (0.001), S1507.2 (0.003)]. c) SPP1 knockdown significantly reduced cell invasiveness; crystal violet was used to stain invasive cells. Cells were then eluted with $1 \%(w / v)$ SDS, and the absorbance was read at $550 \mathrm{~nm}$ on a Genova MK3 Lifescience Analyser; the number of cells was calculated by plotting the absorbance against a standard curve. The percentage of invasive cells was calculated in three individual experiments. SPP1 knockdown significantly reduced cell invasion in all four MPNST cell lines, suggesting a potential role for SPP1 in metastasis [P values for the four cell lines were as follows: ST8814 (0.0001), S462 (0.001), S1844.1 (0.0001), S1507.2 (0.001). *P < 0.05 when comparing treated vs. untreated cells].

promote the growth of different tumours [38-49]. Cells harbouring activated RAS have been found to exhibit a higher level of SPP1 [50]. It could therefore be that the activation of RAS through the functional loss of neurofibromin (due to NF1 gene inactivation) gives rise to the overexpression of SPP1. Furthermore, the use of SPP1 inhibitors such as agelastatin A has successfully reduced colony formation, migration and invasion in human breast cancer cell lines [51]. Whilst SPP1 has the highest level of differential gene expression, the remaining genes in Table 2 


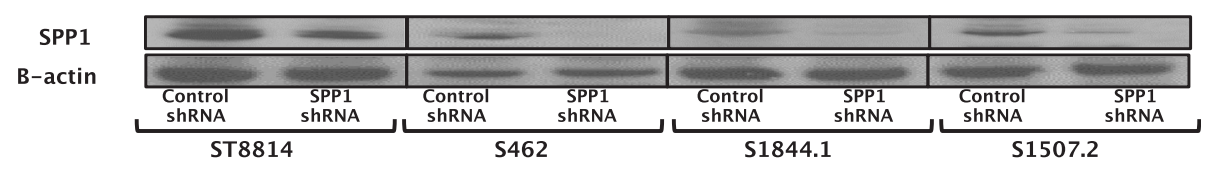

Figure 5 To confirm efficient knockdown, SPP1 protein levels were compared between control and SPP1 knockdown cell lines by Western blot. $\beta$-actin was used as a loading control. SPP1 protein levels were significantly reduced after SPP1 knockdown in all MPNST cell lines.

would warrant further exploration in future studies. This would help to explore further the relationship with NF1 of not only SPP1 but the other identified statistically significant genes, which are differentially expressed between benign and malignant NF1 tumours.

In an attempt to explore the relationship between gene expression and copy number variation in the context of NF1 tumorigenesis, we attempted to integrate previous copy number data derived from the MPNSTs under study here [25], with the newly generated data on differentially expressed genes. The same tumour samples were utilised for both analyses. The identification of prognostic biomarkers for NF1-MPNSTs using gene expression microarrays is challenging in that there are very few candidate genes in common between the different studies so far performed [11-30]. However, over the last 5 years, studies of cancer have begun to integrate gene expression and copy number analysis in an attempt to explore underlying mechanisms of tumorigenesis and to identify potential gene and pathway biomarkers [31,32]. Such an integrative approach has led to advances in our understanding of the role of copy number alterations in tumorigenesis. Thus, for example, the Cancer Genome Atlas project [52] is generating multiple datasets using different platforms (e.g. gene expression and copy number analysis) from the same set of patients. Although the increased expression of a particular gene does not by itself constitute direct evidence for the role of that gene in tumorigenesis, concomitant copy number alterations may serve to disrupt metabolic and physiological processes, thereby contributing indirectly to tumorigenesis $[31,32]$. There is however compelling evidence for a cisdosage effect of CNA on gene expression [53], and this relationship can facilitate the identification of novel genes involved in tumorigenesis as well as other aspects of cancer biology. In any such analysis, it is important to be aware of cellular heterogeneity within the tumour [54].

Work on different tumours, including breast, lung, prostate, hepatocellular carcinoma and melanoma, has yielded an estimate of the proportion of all differentially expressed genes whose expression is concordant with their copy number status. This has been shown to vary between $32 \%$ and $78 \%$ [31,32,55,56]. In this context, it is encouraging that in the current study, $63 \%$ of genes were found to be concordant in terms of their expression and copy number status. However, although SPP1 was found to be the most differentially expressed gene in the initial part of this study, we failed to find any copy number changes in MPNSTs in our earlier study and thus its expression was not concordant with the copy number changes [25]. For this reason, we propose that the observed upregulation of SPP1 gene expression may not have been modulated by a copy number alteration. This is especially in view of the fact that no copy number alterations involving SPP1 have previously been reported in a variety of cancers and tissue types (by reference to COSMIC and CONAN) [57,58], including NF1 MPNSTs [40]. Thus, in the absence of gene duplication/amplification/deletion, it may be that there are other genetic mechanisms including DNA methylation, point mutations, up- or downregulated transcription factors, regulation of messenger RNA (mRNA) transcription or microRNAs (miRNAs) [59-61] that could influence the expression of the non-concordant genes (37\%) identified in this study.

Finally, we performed pathway analysis on the 76 genes that were common to the copy number array and exon array data. Integration of clinical information with copy number and gene expression data has led to the identification of genes common to CNA regions and expression array datasets that are consistently associated with clinical outcomes including lung cancer, thereby underlining the clinical relevance and utility of such data sets [56]. The top ten statistically significant pathways were noted from those where two or more genes exhibiting alterations in copy number and/or expression belonged to the same pathway (Table 3). These pathways included glutathione metabolism, Wnt signalling, cell adhesion PLAU and intracellular signalling by alpha6/beta-4 (a6b4) integrins. The Wnt signalling pathway, including the genes CSNK1D and DAB2 in this data set (Table 3), is of particular interest. The $A P C$ gene belongs to the Wnt pathway and is somatically mutated in various cancers and also in familial adenomatous polyposis, which results from inherited $A P C$ gene mutations [62]. In human colon cancer, SPP1 is a transcriptional target of aberrant Wnt signalling, and SPP1 expression alone predicts survival [63]. Vinas et al. (2010) demonstrated that the antiapoptotic role of Wnt was mediated by SPP1, a direct Wnt target gene, and SPP1 was reduced by Wnt antibody administration in vivo [64]. Using comparative transcriptome analysis, Mo et al. (2013) previously demonstrated 
that PI3K and $\beta$-catenin signalling are involved in the promotion of MPNST growth [26]. Furthermore, using a sleeping beauty forward genetic screen, the Wnt pathway has been found to be a driver of MPNST development [27]. Most interestingly, in a recent study, $20 \mathrm{Wnt}$ genes exhibited altered expression in MPNST biopsies and cell lines in comparison to benign neurofibromas [30]. Taken together, it has become clear that progression to malignancy requires many genomic alterations acting in concert. Importantly, our results appear to concur with the findings of previous studies that the canonical Wnt signalling pathway is likely to be a key driver of MPNST development. Thus, members of the Wnt pathway may not only constitute potential biomarkers of MPNST tumorigenesis but also represent potential therapeutic targets for small molecule inhibitors [26-28].

In addition to the Wnt signalling pathway, other pathways in Table 3 are also thought to be involved in cancer progression. Specifically, although there is no clear association reported with NF1, altered glutathione (GSH) metabolism is thought to be a major mechanism of chemoresistance and GSH levels are reportedly elevated in non-small cell lung cancer [65]. In addition, genetic variations in genes involved in the glutathione and DNA repair pathways are associated with non-small cell lung cancer survival [66]. Further analysis of the pathways in Table 3 would be important to ascertain the role of the $N F 1$ gene in other cancers.

\section{Conclusions}

Although MPNSTs only develop in approximately $15 \%$ of NF1 patients, they represent a frequent cause of lethal progression of the NF1 phenotype. It is clear that many genetic (and potentially epigenetic) factors contribute to abnormal tumour progression in these neoplasms $[67,68]$. The prognosis for individuals diagnosed with an MPNST is usually very poor, especially as treatment options for MPNSTs are currently very limited and complete surgical excision with clear margins has proved to be very difficult. In knockdown experiments involving shRNA for SPP1, we found that cell migration was reduced in four different MPNST cell lines. This exploratory study supports the idea of a direct role for SPP1 in MPNST development and metastasis. Although obtaining MPNST samples can be challenging, this preliminary study warrants confirmation in a larger panel of samples as the study has a limitation in that it is based on a small cohort of samples. In addition to the well-studied role of osteopontin in other cancers [38-49], the expression of SPP1 is regulated by Wnt signalling, one of the pathways that we identified as playing a role in the progression of benign plexiform neurofibromas to MPNSTs [69]. This is most encouraging in terms of the potential for those genes and pathways newly identified in this study to help in understanding the molecular basis of tumorigenesis and malignant transformation in NF1 as well as providing targets for therapeutic intervention in NF1-MPNST development.

\section{Methods}

\section{Patient samples and RNA preparation}

DNA and RNA were isolated from the same segment of tumour. RNA was extracted from nine NF1-associated tumours with biallelic NF1 gene mutations (comprising four benign PNFs and five high grade MPNSTs) from nine unrelated NF1 patients using the TRIZOL method (Invitrogen) as previously described [25]. The same tumour samples were utilised in the current study and the previous analyses for microarray analysis [25] to enable analysis of paired CNA and gene expression data. All total RNA samples were assessed for purity and integrity by means of an Agilent Bioanalyser. Samples were renamed and randomised in order to avoid order bias or batching effects. Analysis of gene expression by Affymetrix Human Exon 1.0 ST Array was performed by Almac Diagnostics (Craigavon, UK). The Affymetrix Human Exon 1.0 ST array contains 17,881 transcripts. Standard operating protocols, as provided by the manufacturer, were used to PCR amplify and hybridise nine NF1associated tumour-derived cDNA samples on Affymetrix Human Exon 1.0 ST arrays.

\section{Ethics statement}

All patients provided informed consent and the study was approved by the Research Ethics Committee (REC) for Wales and appropriate institutional review boards.

\section{Identification of differentially expressed genes between benign neurofibromas and MPNSTs}

A quality control (QC) assessment of the Exon array profiles was performed to examine standard Affymetrix quality parameters, expression distribution patterns and array profile relationships. Overall, a high quality level was achieved (median percent present $=65.99 \%$ ), although the average expression level of all probe sets across the array profiles was quite variable. "percent present" represents the proportion of probe sets 'present' or detected as defined by the DABG (detection above background) algorithm. The DABG algorithm yields a detection metric ( $P$ value) generated by comparing perfect match probes to a distribution of background probes. A probe set was considered to be 'present' if the DABG $P$ value was $\leq 0.01$. Further information is available at www.affymetrix.com. In an attempt to mitigate the variable expression profile observed, differential gene expression analysis was performed with and without those samples from either end of the observed expression range, namely S0342F0011 (malignant), S0342F0020 (malignant) and S0342F0030 (benign). The results that were common to both differential 
analyses were taken forward for further analysis. Probe-level data were analysed using the robust multi-array average (RMA) method to generate gene-level measurements [70].

The expression levels of all genes on the array were compared between benign PNFs and MPNSTs, at two different levels of stringency. Under the less stringent criteria, statistical testing employed a $P$ value threshold of 0.05 , which served to identify genes displaying 1.3 fold or more (absolute) differential gene expression. The two levels of stringency that were used as fold change cut-offs, in this sense, were essentially arbitrary but nevertheless provide an element of choice in terms of prioritising targets to follow up. We have filtered at 1.3, which may be interpreted as "low stringency". However, combined with a significant $P$ value, this can often be valid in the context of those biological systems where some changes are expected to be subtle yet functionally relevant. Moreover, although the fold change estimations from microarray data compared to qPCR are frequently underestimated, it is often possible to validate findings by qPCR from the lower fold change space of microarray data. Under high stringency criteria, statistical testing employed a $P$ value threshold of 0.005 , which was deemed to be sufficient to identify genes displaying 1.5 -fold or more (absolute) differential gene expression. This approach allowed us to interrogate two different sets of expression data, thereby minimising the loss of important information.

\section{Quantitative real-time PCR (q-PCR)}

All of the top 20 differentially expressed genes were independently assessed by quantitative PCR using RNA isolated from additional MPNST samples, not used in the previous or current study. Due to the small initial sample size, we felt that it was necessary to determine the validity of the top 20 genes in an independent cohort of samples. These 20 genes, plus the $B 2 M$ [NCBI: NM_004048] endogenous control identified in our analysis were: FAP [NCBI: NM_ 004460], NSBP1 [NCBI: NM_030763], KRTAP13-4 [NCBI: NM_181600], PTGIS [NCBI: NM_000961], KIAA0746 [NCBI: NM_015187], SPP1 [NCBI: NM_001040058], FAM177A1 [NCBI: NM_001079519], ADH1B [NCBI: NM_000668], GRIK3 [NCBI: NM_000831], FLJ42200 [NCBI: AK124194], COL10A1 [NCBI: NM_000493], CSRP1 [NCBI: NM_004078], GRIK2 [NCBI: NM_175768], CD72 [NCBI: NM_001782], EFHC2 [NCBI: NM_025184], SLC25A12 [NCBI: NM_003705], GTDC1 [NCBI: NM_ 001006636], PEG3 [NCBI: NM_006210], CCT5 [NCBI: NM_012073] and TDP1 [NCBI: NM_018319]. qPCR was performed as previously described [25]. Primers for all genes are listed in Additional file 4: Table S1.

\section{Cell lines and maintenance}

ST8814 MPNST-derived cell lines were purchased from ATCC (distributed by LGC Standards, Middlesex, UK).
The S462, S1507.2 and S1488.1 MPNST cell lines were a kind gift from Prof. Mautner (University of Hamburg, Germany) and the late Prof. Guha (University of Toronto, Canada). After SPP1 knockdown, cell lines were cultured and maintained in Dulbecco's Modified Eagle Medium (DMEM) supplemented with 10\% (v/v) foetal bovine serum (FBS) and $1 \%(\mathrm{v} / \mathrm{v})$ penicillin-streptomycin and $5 \mathrm{ng} / \mathrm{ml}$ puromycin in a humidified incubator $\left(5 \% \mathrm{CO}_{2}\right.$ at $\left.37^{\circ} \mathrm{C}\right)$.

\section{Lentivirus generation and shRNA knockdown of SPP1}

Both SPP1 shRNA (Clone ID: NM_000582.2-597s1c1) and non-target control MISSION shRNA (Clone ID: SHCO16) in pLKO.1 vector (Sigma-Aldrich Company Ltd., Dorset, UK) were packaged into lentivirus using HEK293T cells co-transfected (lipofectamine 2000, Life Technology, Paisley, UK) with pLP1, pLP2 and pLP (VSVG). Confluent MPNST cell lines were infected with shRNA-containing lentivirus (SPP1 or non-target control) and selected over 2 weeks with $5 \mu \mathrm{g} / \mathrm{ml}$ puromycin (Life Technology, Paisley, UK). Puromycin-selected mixed cell populations were then used for tumour formation, wound healing and invasion assays.

\section{Tumour spheroid assays}

Two-layered soft agar assays were carried out in six-well plates. MPNST cell lines were plated in complete DMEM media in $0.3 \%(\mathrm{v} / \mathrm{v})$ agar at a density of $3 \times 10^{5} \mathrm{cells} / \mathrm{mL}$ over a $0.6 \%(\mathrm{v} / \mathrm{v})$ agar layer. The agar was then overlaid with complete DMEM media supplemented with $0.1 \mu \mathrm{M}$ puromycin (Life Technology, Paisley, UK). Colonies of MPNSTs were grown for 14 days at $37^{\circ} \mathrm{C}$ in $5 \% \mathrm{CO}_{2}$. Media were changed twice a week. Representative pictures were taken using an inverted AMG EVOS microscope equipped with an Olympus camera. The diameter of tumour spheroids was measured using Image software; the chamber of a haemocytometer $(250 \mu \mathrm{m})$ was used as a scale bar for measurement.

\section{Wound healing}

Cells were seeded in $60-\mathrm{mm}$ plates and left to reach $100 \%$ confluency. Cells were then synchronised in $1 \%$ (v/v) FBS DMEM for $24 \mathrm{~h}$ and "wounded" with a pipette tip. Dead cells were removed with PBS wash and then replaced with DMEM (10\% (v/v) FBS). Pictures were taken at 0 and $12-18 \mathrm{~h}$ using an inverted AMG EVOS microscope equipped with an Olympus camera.

\section{Invasion assays}

Transwell permeable supports with 6.5-mm diameter inserts, $8.0-\mu \mathrm{m}$ pore size and a polycarbonate membrane (Corning, cat no: 3428) were used to perform the invasion assays. Cells were grown in a $75-\mathrm{cm}^{2}$ flask with 
standard medium (10\% (v/v) FBS) until confluent. Cells were then harvested using Trypsin-EDTA. Cells were counted using a haemocytometer; $1 \times 10^{6}$ cells were resuspended in DMEM containing 1\% (v/v) FBS. The top chamber of the Transwell was filled with $300 \mu \mathrm{l}$ BD Matrigel basement membrane matrix $(1 \mathrm{mg} / \mathrm{ml})$. The Matrigel was incubated at $37^{\circ} \mathrm{C}$ for $4 \mathrm{~h}$ to allow it to gel. Cells were then seeded in the upper chamber of the Transwell; the lower chamber was filled with $600 \mu \mathrm{l}$ standard culture medium (10\% (v/v) FBS) and $5 \mathrm{mg} / \mathrm{mL}$ fibronectin (R\&D systems, Abingdon, UK), as an adhesive substrate. Cells were incubated at $37^{\circ} \mathrm{C} 5 \% \mathrm{CO}_{2}$ for 3 days. The proportion of adherent cells was then determined by fixing the cells with methanol and acetone (1:1) for 20 min at $-20^{\circ} \mathrm{C}$. Cells were then stained with crystal violet $(5 \mathrm{mg} / \mathrm{ml})$ in ethanol for $10 \mathrm{~min}$, followed by a stringent wash with $\mathrm{dH}_{2} \mathrm{O}$ until the water ran clear. Crystal violetstained cells were eluted with $1 \%(\mathrm{w} / \mathrm{v})$ SDS and the absorbance read at $550 \mathrm{~nm}$ on a Genova MK3 Life Science Analyser (Jenway Scientific, Staffordshire, UK). Three replicates were performed per cell line to enable statistical analysis.

\section{Western blotting}

The NuPage Novex gel system was used for electrophoresis as described in the manufacturer's protocol (Life Technology, Paisley, UK). Protein samples were resolved on $4 \%-12 \%$ Bis-Tris gels to identify a band size of 66 $\mathrm{kD}$. Proteins were then transferred to a polyvinylidene fluoride (PDVF) membrane purchased from Millipore U.K. Ltd. (Watford, UK), blocked in 5\% (w/v) dry milk powder in standard Tris-buffer saline supplemented with $0.1 \%(\mathrm{v} / \mathrm{v})$ Tween [as recommended by Cell Signalling Technology Inc. (Danvers, MA, USA)] for $4 \mathrm{~h}$. Membranes were incubated at $4^{\circ} \mathrm{C}$ overnight in primary antibody (1:200 dilutions in 2\% (w/v) BSA in TBS-T) then washed twice for $4 \mathrm{~min}$ in TBS-T and incubated in the appropriate HRP-conjugated secondary antibody $(1: 10,000$ dilution in $5 \%(\mathrm{w} / \mathrm{v})$ Marvel in TBS-T) for a minimum of $30 \mathrm{~min}$ at room temperature. Membranes were washed four times for 3 min with TBS-T and then incubated in enhanced chemiluminescence (ECL) solution (GE Healthcare Life Sciences, Buckinghamshire, UK) for $1 \mathrm{~min}$. Konica Medical Film was used to visualise the signal, and the exposed films were developed using a Konica Minolta SRX-101A developer.

\section{Statistical analysis}

SPSS software was used for the statistical analysis of tumour spheroid formation $(n=40)$ and the wound healing $(n=3)$ and invasion $(n=3)$ assays. A one-way ANOVA was performed on the data sets to obtain the requisite $P$ values.

\section{Additional files}

Additional file 1: Table S2. The four distinct sets of genes identified from the various analyses that were performed of differentially expressed genes.

Additional file 2: Table S3. Data on copy number alterations compared with the genes found to be differentially expressed between MPNSTs and PNFs. 76/121 genes exhibited concordance between copy number changes and the level of expression.

Additional file 3: Figure S1. Results of the q-PCR analysis in relation to the top 20 genes differentially expressed between MPNSTs and PNFs.

Additional file 4: Table S1. Primers used for $q-P C R$ analysis and results of the q-PCR analysis.

\section{Abbreviations}

CNA: copy number alteration; DABG: detection above background; HMM: hidden Markov model; MPNST: malignant peripheral nerve sheath tumour; NF1: neurofibromatosis type 1; PNF: peripheral neurofibroma; shRNA: short hairpin RNA.

\section{Competing interests}

The authors declare that they have no competing interests.

\section{Authors' contributions}

LET completed the molecular genetic studies, data analysis, coordination of the project and drafting of the manuscript. JW completed the QPCR assays. EMR carried out the cell assays. MM performed the statistical analysis. KMD completed the wWestern blot analysis. ART created the lentiviral vector and assisted with the cell assays and western blots. FM and SM completed the microarray work as part of Almac Diagnostics. DNC helped to draft the manuscript and provided guidance. MU conceived of the study, participated in its design and coordination and helped to draft the manuscript.

All authors read and approved the final manuscript.

\section{Acknowledgements}

We are grateful to Professor Victor Mautner and the late Professor Guha for providing tumour samples. We also thank Chris Lovejoy for his technical support and the lan Owen Trust and the Wales Gene Park for their financial support. We are also grateful to Dr lan Frayling for his encouragement.

\section{Author details}

${ }^{1}$ Institute of Medical Genetics, School of Medicine, Cardiff University, Cardiff CF14 4XN, UK. ${ }^{2}$ Almac Diagnostics, 19 Seagoe Industrial Estate, Craigavon, Northern Ireland BT63 5QD, UK.

Received: 14 November 2014 Accepted: 18 January 2015

Published online: 15 February 2015

\section{References}

1. Huson S. The neurofibromatoses: classification, clinical features and genetic counselling. In: Kaufmann D, editor. Neurofibromatoses (monographs in human genetics), vol. 16. Switzerland: S Karger AG; 2008. p. 1-20.

2. Upadhyaya M. Genetic basis of tumorigenesis in NF1 malignant peripheral nerve sheath tumors. Front Biosci. 2011;16:937-51.

3. Ferner RE, Gutmann DH. International consensus statement on malignant peripheral nerve sheath tumours in neurofibromatosis 1. Cancer Res. 2002;1(62(5)):1573-7.

4. Evans DG, Baser ME, McGaughran J, Sharif S, Howard E, Moran A. Malignant peripheral nerve sheath tumours in neurofibromatosis 1. J Med Genet. 2002;9:311-4.

5. Katz D, Lazar A, Lev D. Malignant peripheral nerve sheath tumour (MPNST): the clinical implications of cellular signalling pathways. Expert Rev Mol Med. 2009;11:e30.

6. Tucker T, Wolkenstein P, Revuz J, Zeller J, Friedman J. Association between benign and malignant peripheral nerve sheath tumors in NF1. Neurology. 2005;65:205-11.

7. Mautner VF, Kluwe L, Friedrich RE, et al. Clinical characterisation of 29 neurofibromatosis type-1 patients with molecularly ascertained $1.4 \mathrm{Mb}$ type-1 NF1 deletions. J Med Genet. 2010;47:623-30. 
8. Ferner RE, Hughes RA, Hall SM, Upadhyaya M, Johnson MR. Neurofibromatous neuropathy in neurofibromatosis 1 (NF1). J Med Genet. 2004:41:837-41.

9. Evans DG, Huson SM, Birch JM. Malignant peripheral nerve sheath tumours in inherited disease. Clin Sarcoma Res. 2012;2:17.

10. Beert $\mathrm{E}$, Brems $\mathrm{H}$, Daniëls $\mathrm{B}$, et al. Atypical neurofibromas in neurofibromatosis type 1 are premalignant tumors. Genes Chrom Cancer. 2011;50:1021-32.

11. Lothe RA, Karhu R, Mandahl N, et al. Gain of 17q24-qter detected by comparative genomic hybridization in malignant tumors from patients with von Recklinghausen's neurofibromatosis. Cancer Res. 1996;56:4778-81.

12. Bridge Jr RS, Bridge JA, Neff JR, Naumann S, Althof P, Bruch LA. Recurrent chromosomal imbalances and structurally abnormal breakpoints within complex karyotypes of malignant peripheral nerve sheath tumour and malignant triton tumour: a cytogenetic and molecular cytogenetic study. J Clin Pathol. 2004;57:1172-8.

13. Watson MA, Perry A, Tihan $T$, et al. Gene expression profiling reveals unique molecular subtypes of neurofibromatosis type I-associated and sporadic malignant peripheral nerve sheath tumors. Brain Pathol. 2004;14:297-303.

14. Storlazzi CT, Brekke HR, Mandahl N, et al. Identification of a novel amplicon at distal 17q containing the BIRC5/SURVIVIN gene in malignant peripheral nerve sheath tumours. J Pathol. 2006;209:492-500.

15. Shen MH, Mantripragada K, Dumanski JP, Frayling I, Upadhyaya M. Detection of copy number changes at the NF1 locus with improved high-resolution array CGH. Clin Genet. 2007;72:238-44.

16. Kresse SH, Skårn M, Ohnstad HO, et al. DNA copy number changes in high-grade malignant peripheral nerve sheath tumors by array $\mathrm{CGH}$. Mol Cancer. 2008;7:48.

17. Mantripragada KK, Spurlock G, Kluwe L, et al. High-resolution DNA copy number profiling of malignant peripheral nerve sheath tumors using targeted microarray-based comparative genomic hybridization. Clin Cancer Res. 2008;14:1015-24.

18. Mantripragada KK, Díaz de Ståhl T, Patridge C, et al. Genome-wide high-resolution analysis of DNA copy number alterations in NF1-associated malignant peripheral nerve sheath tumors using $32 \mathrm{~K} \mathrm{BAC}$ array. Genes Chrom Cancer. 2009:48:897-907.

19. Brekke HR, Kolberg M, Skotheim Rl, et al. Identification of p53 as a strong predictor of survival for patients with malignant peripheral nerve sheath tumors. NeuroOncol. 2009;11:514-28.

20. Miller SJ, Jessen WJ, Mehta T, et al. Integrative genomic analyses of neurofibromatosis tumours identify SOX9 as a biomarker and survival gene. EMBO Mol Med. 2009;1:236-48.

21. Pemov A, Park C, Reilly KM, Stewart DR. Evidence of perturbations of cell cycle and DNA repair pathways as a consequence of human and murine NF1-haploinsufficiency. BMC Genomics. 2010;11:194.

22. Brekke HR, Ribeiro FR, Kolberg M, et al. Genomic changes in chromosomes 10,16 , and $X$ in malignant peripheral nerve sheath tumors identify a high-risk patient group. J Clin Oncol. 2010;28:1573-82.

23. Subramanian S, Thayanithy $\mathrm{V}$, West RB, et al. Genome-wide transcriptome analyses reveal p53 inactivation mediated loss of miR-34a expression in malignant peripheral nerve sheath tumours. J Pathol. 2010;220:58-70.

24. Chai G, Liu N, Ma J, et al. MicroRNA-10b regulates tumorigenesis in neurofibromatosis type 1. Cancer Sci. 2010;101:1997-2004.

25. Upadhyaya M, Spurlock G, Thomas L, et al. Microarray-based copy number analysis of neurofibromatosis type-1 (NF1)-associated malignant peripheral nerve sheath tumors reveals a role for Rho-GTPase pathway genes in NF1 tumorigenesis. Hum Mutat. 2012;33:763-76.

26. Mo W, Chen J, Patel A, et al. CXCR4/CXCL12 mediate autocrine cell-cycle progression in NF1-associated malignant peripheral nerve sheath tumors. Cell. 2013:152:1077-90.

27. Rahrmann EP, Watson AL, Keng WW, et al. Forward genetic screen for malignant peripheral nerve sheath tumor formation identifies new genes and pathways driving tumorigenesis. Nat Genet. 2013;45:756-66.

28. Watson AL, Rahrmann EP, Moriarity BS, et al. Canonical Wnt/ $\beta$-catenin signaling drives human Schwann cell transformation, progression, and tumor maintenance. Cancer Discov. 2013;6:674-89.

29. Largaespada D, Ratner N. Interweaving the strands: $\beta$-catenin, an HIV co-receptor, and Schwann cell tumors. Cancer Cell. 2013;23:269-71.

30. Luscan A, Shackleford G, Masliah-Planchon J, et al. The activation of the WNTWnt signalling pathway is a hallmark in Neurofibromatosis type 1 tumorigenesis. Clin Cancer Res. 2013;15(20(2)):358-71.
31. Huang N, Shah PK, Li C. Lessons from a decade of integrating cancer copy number alterations with gene expression profiles. Brief Bioinformatics. 2012;13:305-16.

32. Hassan A, Mokhtar NM, Sin K, Mohammed Rose I, Sagap I, Harun R, et al. Integrated analysis of copy number variation and genome-wide expression profiling in colorectal cancer tissues. PLoS ONE. 2014;2(9(4)):e92553.

33. Rittling SR, Chambers AF. Role of osteopontin in tumour progression. Brit J Cancer. 2004;90:1877-81.

34. Pradervand S, Paillusson A, Thomas J, et al. Affymetrix Whole-Transcript Human Gene1.0 ST array is highly concordant with standard 3' expression arrays. Biotechniques. 2008:44:759-62.

35. Xi L, Feber A, Gupta V, et al. Whole genome exon arrays identify differential expression of alternatively spliced, cancer-related genes in lung cancer. Nucleic Acids Res. 2008;36:6535-47.

36. Thorsen K, Sørensen KD, Brems-Eskildsen AS, et al. Alternative splicing in colon, bladder, and prostate cancer identified by exon array analysis. Mol Cell Proteomics. 2008;7:1214-24.

37. Kapur K, Xing Y, Ouyang Z, Wong WH. Exon arrays provide accurate assessments of gene expression. Genome Biol. 2007:8:R82.

38. Furger KA, Menon RK, Tuck AB, Bramwell VH, Chambers AF. The functional and clinical roles of osteopontin in cancer and metastasis. Current Mol Med. 2001;1:621-32.

39. Bramwell VHC, Tuck AB, Wilson SM, et al. Expression of osteopontin and HGF/met in adult soft tissue tumors. Cancer Biol Ther. 2005;4:1336-41.

40. Hoshi N, Sugino T, Suzuki T. Regular expression of osteopontin in granular cell tumor: distinctive feature among Schwannian cell tumors. Pathol Int. 2005;55:484-90.

41. Rangaswami H, Bulbule A, Kundu G. Osteopontin: role in cell signaling and cancer progression. Trends Cell Biol. 2006;16:79-87.

42. Wai PY, Kuo PC. Osteopontin: regulation in tumor metastasis. Cancer Metastasis Rev. 2008;27:103-18.

43. Weber GF. The metastasis gene osteopontin: a candidate target for cancer therapy. Biochim Biophys Acta. 2001;1552:61-85.

44. Shevde LA, Das S, Clark DW, Samant RS. Osteopontin: an effector and an effect of tumor metastasis. Curr Mol Med. 2010;10:71-81.

45. Ahmed M, Behera R, Chakraborty G, et al. Osteopontin: a potentially important therapeutic target in cancer. Expert Opin Ther Targets. 2011;15:1113-26.

46. Luo X, Ruhland MK, Pazolli E, Lind AC, Stewart SA. Osteopontin stimulates preneoplastic cellular proliferation through activation of the MAPK pathway. Mol Cancer Res. 2011;9:1018-29.

47. Rao G, Du L, Chen Q. Osteopontin, a possible modulator of cancer stem cells and their malignant niche. Oncoimmunology. 2013;2:e24169.

48. El-Tanani MK, Campbell FC, Kurisetty V, Jin D, McCann M, Rudland PS. The regulation and role of osteopontin in malignant transformation and cancer. Cytokine Growth Factor Rev. 2006;17:463-74.

49. Tilli TM, Mello KD, Ferreira LB, et al. Both osteopontin-c and osteopontin-b splicing isoforms exert pro-tumorigenic roles in prostate cancer cells. Prostate. 2012;72:1688-99.

50. Guo X, Zhang YP, Mitchell DA, Denhardt DT, Chambers AF. Identification of a Ras-activated enhancer in the mouse osteopontin promoter and its interaction with a putative ETS-related transcription factor whose activity correlates with the metastatic potential of the cell. Mol Cell Biol. 1995;15:476-87.

51. Mason CK, McFarlane S, Johnston PG, et al. Agelastatin A: a novel inhibitor of osteopontin-mediated adhesion, invasion, and colony formation. Mol Cancer Ther. 2008;7(3):548-58.

52. The cancer genome atlas [http://cancergenome.nih.gov]

53. Gu W, Choi H, Ghosh D. Global associations between copy number and transcript mRNA microarray data: an empirical study. Cancer Inform. 2008:6:17-23.

54. Thomas L, Mautner VF, Cooper DN, Upadhyaya M. Molecular heterogeneity in malignant peripheral nerve sheath tumors associated with neurofibromatosis type 1. Hum Genomics. 2012;4(6):18.

55. Spivey $T L$, De Giorgi $V$, Zhao $Y$, et al. The stable traits of melanoma genetics: an alternate approach to target discovery. BMC Genomics. 2012;13:156.

56. Lu TP, Lai LC, Tsai MH, et al. Integrated analyses of copy number variations and gene expression in lung adenocarcinoma. PLoS ONE. 2011;6:e24829.

57. Conan copy number analysis [http://www.sanger.ac.uk/research/projects/ cancergenome/copy_number.html]

58. COSMIC catalogue of somatic mutations in cancer [http://cancer.sanger.ac. uk/cancergenome/projects/cosmic] 
59. Kleinjan DA, Lettice LA. Long-range gene control and genetic disease. Adv Genet. 2008;1:339-88.

60. Henrichsen CN, Chaignat E, Reymond A. Copy number variants, diseases and gene expression. Hum Mol Genet. 2009;18(R1):R1-8.

61. Hyman E, Kauraniemi P, Hautaniemi S, et al. Impact of DNA amplification on gene expression patterns in breast cancer. Cancer Res. 2002;62:6240-5.

62. White BD, Chien AJ, Dawson DW. Dysregulation of Wnt/ $\beta$-catenin signaling in gastrointestinal cancers. Gastroenterology. 2012;142:219-32.

63. Reilly KM. Extending the convergence of canonical Wnt signaling and classic cancer pathways for treatment of malignant peripheral nerve sheath tumors. Cancer Discov. 2013;6:610-2.

64. Vinas JL, Sola A, Jung M, Mastora C, Vinuesa E, Pi F, et al. Inhibitory action of Wnt target gene osteopontin on mitochondrial cytochrome c release determines renal ischemic resistance. Am J Physiol Renal Physiol. 2010;299:F234-42.

65. Blair SL, Heerdt P, Sachar S, et al. Glutathione metabolism in patients with non-small cell lung cancers. Cancer Res. 1997;57:152-5.

66. Sun Z, Chen J, Aakre J, et al. Genetic variation in glutathione metabolism and DNA repair genes predicts survival of small-cell lung cancer patients predicts survival of small-cell lung cancer patients. Ann Oncol. 2011:21:2011-6.

67. Harder A, Titze S, Herbst L, Harder T, Guse K, Tinschert S, et al. Monozygotic twins with neurofibromatosis type 1 (NF1) display differences in methylation of NF1 gene promoter elements, $5^{\prime}$ untranslated region, exon and intron 1. Twin Res Hum Genet. 2010;13(6):582-94

68. Titze S, Peters H, Währisch S, Harder T, Guse K, Buske A, et al. Differential MSH2 promoter methylation in blood cells of neurofibromatosis type 1 (NF1) patients. Eur J Hum Genet. 2010;18(1):81-7.

69. Rohde F, Rimcus $C$, Friederichs J, et al. Expression of osteopontin, a target gene of de-regulated Wnt signalling predicts survival of colon cancer. Int J Cancer. 2007:121:1717-23.

70. Irizarry RA, Hobbs B, Collin F, et al. Exploration, normalization, and summaries of high density oligonucleotide array probe level data. Biostatistics. 2003;4:249-64.

\section{Submit your next manuscript to BioMed Central and take full advantage of:}

- Convenient online submission

- Thorough peer review

- No space constraints or color figure charges

- Immediate publication on acceptance

- Inclusion in PubMed, CAS, Scopus and Google Scholar

- Research which is freely available for redistribution 